\title{
Double Operator Integrals and Submajorization
}

\author{
D. Potapov and F. Sukochev* \\ School of Mathematics and Statistics, University of NSW, \\ Kensington NSW 2052, Australia
}

\begin{abstract}
We present a user-friendly version of a double operator integration theory which still retains a capacity for many useful applications. Using recent results from the latter theory applied in noncommutative geometry, we derive applications to analogues of the classical Heinz inequality, a simplified proof of a famous inequality of Birman-Koplienko-Solomyak and also to the ConnesMoscovici inequality. Our methods are sufficiently strong to treat these inequalities in the setting of symmetric operator norms in general semifinite von Neumann algebras.
\end{abstract}

Key words: double operator integration, unitarily invariant norm inequalities, noncommutative $L_{p}$-spaces

AMS subject classification: 46L51, 46L52, 47L20.

\section{Introduction}

The notion of a double operator integral emerged from the papers of Yu.L. Daletskii and S.G. Krein [7], [8]. Such an integral is formally written as

$$
Q(T)=\iint \phi(\lambda, \mu) d F_{\mu} T d E_{\lambda}
$$

where $E_{\lambda}, F_{\mu}$ are spectral families in the separable Hilbert space $\mathcal{H}, T$ is some bounded linear operator on $\mathcal{H}$, and $\phi(\lambda, \mu)$ is a bounded complex-valued function, where the integral is considered in

*Corresponding author. E-mail: f.sukochev@unsw.edu.au. The work is partially supported by the ARC. 
the sense of iterated Riemann-Stieltjes integration. Further development of this construction was given in a series of papers by M.Š. Birman and M.Z. Solomyak [4], [5], [6], where, in particular, interesting and important perturbation results for operators in Schatten ideals $\mathfrak{S}^{p}, 1 \leq p \leq \infty$, are given. In the setting of operator ideals (and more generally symmetric spaces of measurable operators affiliated with a general semifinite von Neumann algebra), double operator integration theory has been recently developed in $[18,16,17,23]$. It is probably fair to say that the theory presented in the papers cited above is somewhat technical and not user friendly. Recently, an attempt was made in [19] to present a "baby"(finite-dimensional) version of double operator integration essentially due to Löwner. Our exposition here continues this line of thought. Although we work with infinite-dimensional von Neumann algebras, we have intentionally narrowed here the class of the functions $\phi(\lambda, \mu)$ to which the integration process (1.1) is applicable. This reduction allows us to give a very concise and at the same time fairly complete treatment of the theory, which is still sufficient for applications. In particular, we think that readers familiar with substantial technical difficulties occurring in $[6,16]$ devoted to differentiation of operator-functions would appreciate our approach (and the proof) given in Lemma 7.

It is further important to point out that our current approach is strongly motivated by applications of double operator integration theory to noncommutative geometry given recently in $[2,24,9]$. In particular, Lemma 1 below can be traced to [2] where a somewhat similar approach was suggested to multiple operator integration theory. Furthermore, some technical (double operator integration) instruments developed in [24,9] to treat various commutator and Lipschitz estimates occurring in noncommutative geometry appear to have a wider area of applicability. In the present paper, we show how these instruments can be applied to unitarily invariant norm inequalities. We have decided to illustrate these applications on the classical Heinz inequality, on the famous inequality of (Ando-)Birman-Koplienko-Solomyak and also on the example of one inequality of Connes and Moscovici, which is useful in noncommutative geometry. Even though our approach does not yield the best constants in the first two cases, it is remarkable that all of the above can be treated in a unified manner. It should be mentioned that the idea to use double operator integration techniques in the general area of operator inequalities is certainly not new, it has been around from the very inception of the theory. In particular, the original proof of BirmanKoplienko-Solomyak inequality given in [3] depends on deep facts from double operator integration theory. Our contribution here is of purely technical character and consists mainly in substantial simplification and streamlining of the proof. It is also rather obvious that potential applicability of our method is not exhausted by the three inequalities above; in the text below, we indicate (albeit briefly) some other analogous (and apparently, new) results following from our approach.

\section{Terminology and preliminaries}

Suppose $\mathcal{M}=M_{n}(\mathbb{C})$ is the von Neumann algebra of all $n \times n$ complex matrices. Beginning from the fundamental paper of J. von Neumann [30], it has become customary to consider unitarily invariant norms on $\mathcal{M}$. The class of such norms includes the usual operator norm as well as all Schatten norms. A special class of such norms is given by the (so-called) Ky Fan norms $\|\cdot\|_{(k)}$, 
defined by setting

$$
\|x\|_{(k)}=\sum_{j=1}^{k} s_{j}(x), \quad 1 \leq k \leq n
$$

for any $x \in M_{n}(\mathbb{C})$. Here, $\left\{s_{j}(x)\right\}_{j=1}^{n}$ denotes the singular value sequence of $x$, that is, the sequence of eigenvalues of $|x|=\sqrt{x^{*} x}$ arranged in decreasing order and repeated according to multiplicity. The importance of this class of norms lies in the well-known Ky Fan dominance principle that, for all $x, y \in M_{n}(\mathbb{C})$, the inequality $\mid\|x\|\|\leq\|\|y\|$ holds for any unitarily invariant norm \|\|$\cdot \|$ on $\mathcal{M}$ if and only if the inequality $\|x\|_{(k)} \leq\|y\|_{(k)}$ holds for all $1 \leq k \leq n$. If $x, y \in$ $M_{n}(\mathbb{C})$, then $x$ is said to be submajorised by $y$, written $x \prec \prec y$, if and only if $\|x\|_{(k)} \leq\|y\|_{(k)}$ holds for all $1 \leq k \leq n$, or, equivalently,

$$
\sum_{j=1}^{k} s_{j}(x) \leq \sum_{j=1}^{k} s_{j}(y), \quad 1 \leq k \leq n .
$$

The theory of unitarily invariant operator ideals in the case when the von Neumann algebra $\mathcal{M}$ coincides with the algebra $B(\mathcal{H})$ of all bounded linear operators on a separable Hilbert space $\mathcal{H}$ is very well presented in the existing literature (see e.g. [13, 26]). For an arbitrary compact operator $x \in \mathcal{M}$, the sequence of singular values $\left(s_{n}(x)\right)_{n=1}^{\infty}$ is the sequence of eigenvalues of $|x|=\left(x^{*} x\right)^{1 / 2}$ in decreasing order, repeated according to multiplicity. Given $1 \leq p<\infty$, the Schatten-von Neumann ideal $\mathfrak{S}^{p}$ consists of all compact operators $x \in B(\mathcal{H})$ such that $\left(s_{n}(x)\right)_{n=1}^{\infty}$ falls in the classical Banach sequence space $\ell^{p}$. The norm

$$
\|x\|_{p}:=\left\|\left(s_{n}(x)\right)_{n=1}^{\infty}\right\|_{\ell^{p}}
$$

is a unitarily invariant Banach norm on $\mathfrak{S}^{p}$. If $p=\infty$, then $\mathfrak{S}^{p}$ is identified with $B(\mathcal{H})$ equipped with the uniform operator norm. The definition of submajorization for compact operators remains the same: $x \prec \prec y$ if and only if

$$
\sum_{j=1}^{k} s_{j}(x) \leq \sum_{j=1}^{k} s_{j}(y), \quad 1 \leq k<\infty .
$$

It is a fundamental result in the interpolation theory of linear operators in ideals of compact operators that $x \prec \prec y$ if and only if there exists a linear operator $T: B(\mathcal{H}) \mapsto B(\mathcal{H})$ sending $y$ in $x$ and such that $T$ maps the trace ideal $\mathfrak{S}^{1}$ into itself and $\|T\|_{B(\mathcal{H}) \mapsto B(\mathcal{H})} \leq 1$ and $\|T\|_{\mathfrak{S}^{1} \mapsto \mathfrak{S}^{1}} \leq 1$.

In the case that $\mathcal{M} \subseteq B(\mathcal{H})$ is an arbitrary semifinite von Neumann algebra with normal semifinite faithful (n.s.f.) trace $\tau$, the theory of unitarily invariant operator ideals (and the more general theory of noncommutative symmetric spaces) is strongly related with the theory of noncommutative integration initiated by the pioneering paper of I.Segal [25]. The reader can obtain a quick introduction to the theory of noncommutative $L^{p}$-spaces associated with such algebras as well as a fairly complete exposition of the "state of the art" of that theory from the survey [22]. We denote by $L^{p}=L^{p}(\mathcal{M}, \tau), 1 \leq p \leq \infty$, the corresponding noncommutative $L^{p}$-space. For the treatment of general unitarily invariant (symmetric) operator ideals and the theory of submajorization in the 
setting of semifinite von Neumann algebras, we recommend to the reader the articles [28, 29, 14] and references therein. Finally, in [12], the reader will find a detailed treatment of various other operator inequalities and their connections with submajorization in the setting of general symmetric operator spaces. The approach in [12] and that of the present paper are completely independent of each other.

\section{Double operator integrals}

Let $\phi: \mathbb{R} \times \mathbb{R} \mapsto \mathbb{C}$ be a Borel function admitting the following decomposition

$$
\phi\left(\lambda_{0}, \lambda_{1}\right)=\int_{\Sigma}\left[\prod_{j=0}^{1} \alpha_{j, \sigma}\left(\lambda_{j}\right)\right] d \mu(\sigma),
$$

where $d \mu$ is a complex-valued measure with finite variation $d|\mu|$ on some measure space $(\Sigma, d \mu)$ and where

$$
\alpha_{j, \sigma}: \mathbb{R} \mapsto \mathbb{C}, \quad \sigma \in \Sigma, j=0,1
$$

are bounded Borel functions such that

$$
\int_{\Sigma}\left[\prod_{j=0}^{1}\left\|\alpha_{j, \sigma}\right\|_{\infty}\right] d|\mu|(\sigma)<+\infty .
$$

We denote the class of all functions admitting the representation (3.1) by $\mathfrak{A}$. For every $\phi \in \mathfrak{A}$, we introduce the norm

$$
\|\phi\|_{\mathfrak{A}}:=\inf \int_{\Sigma}\left[\prod_{j=0}^{1}\left\|\alpha_{j, \sigma}\right\|_{\infty}\right] d|\mu|(\sigma),
$$

where the infimum is taken over all possible representations (3.1). The class $\mathfrak{A}$ equipped with the norm $\|\cdot\|_{\mathfrak{A}}$ is a Banach space.

Let $\mathcal{H}$ be a Hilbert space and let $d E_{j}, j=0,1$ be two spectral measures on $\mathbb{R}$ with values in the orthogonal projections in $B(\mathcal{H})$. For every $\phi \in \mathfrak{A}$, we define the operator $T_{\phi}$ on $B(\mathcal{H})$ by

$$
T_{\phi}(x)(\xi)=\int_{\Sigma} A_{0, \sigma} x A_{1, \sigma}(\xi) d \mu(\sigma), \quad \xi \in \mathcal{H}
$$

where $^{\dagger}$

$$
A_{j, \sigma}=\int_{\mathbb{R}} \alpha_{j, \sigma}(\lambda) d E_{j}(\lambda)
$$

and where $\alpha_{j, \sigma}$ and $(\Sigma, d \mu)$ are taken from (3.1), $j=0,1$.

Lemma 1. (i) The operator $T_{\phi}$ above is well-defined, i.e., it does not depend on the choice of representation (3.1).

\footnotetext{
†The integral in (3.2) is a Bochner integral.
} 
(ii) The operator $T_{\phi}$ is bounded if $\phi \in \mathfrak{A}$. Moreover,

$$
\left\|T_{\phi}\right\| \leq\|\phi\|_{\mathfrak{A}} .
$$

Proof. (i) It is sufficient to show that if the bounded Borel functions

$$
\alpha_{j, \sigma}: \mathbb{R} \mapsto \mathbb{C}, \quad \sigma \in \Sigma, j=0,1
$$

satisfy the condition

$$
\int_{\Sigma}\left[\prod_{j=0}^{1} \alpha_{j, \sigma}\left(\lambda_{j}\right)\right] d \mu(\sigma)=0, \quad \lambda_{j} \in \mathbb{R}, \quad j=0,1,
$$

then the operator $T: B(\mathcal{H}) \mapsto B(\mathcal{H})$ defined by

$$
T\left(x_{1}\right)=\int_{\Sigma} A_{0, \sigma} x_{1} A_{1, \sigma} d \mu(\sigma), x_{1} \in B(\mathcal{H}),
$$

where $A_{j, \sigma}, j=0,1$ are given by (3.3), is identically zero. To this end, we shall show that

$$
\operatorname{Tr}\left(x_{0} T\left(x_{1}\right)\right)=0, \quad x_{j} \in B(\mathcal{H}), \quad j=0,1 .
$$

Let us first assume that $x_{j}, j=0,1$ is one-dimensional. Fix vectors $\xi_{j}, \eta_{j} \in \mathcal{H}, j=0,1$ and assume that ${ }^{\ddagger}$

$$
x_{0}=\xi_{1} \otimes \eta_{0} \text { and } x_{1}=\xi_{0} \otimes \eta_{1} .
$$

Fixing $\sigma \in \Sigma$ and using (3.3), we have

$$
\begin{aligned}
\operatorname{Tr}\left(x_{0} A_{0, \sigma} x_{1} A_{1, \sigma}\right)=\prod_{j=0}^{1}\left\langle A_{j, \sigma} \xi_{j}, \eta_{j}\right\rangle & \\
=\prod_{j=0}^{1} \int_{\mathbb{R}} \alpha_{j, \sigma}\left(\lambda_{j}\right)\left\langle d E_{j}\left(\lambda_{j}\right) \xi_{j}, \eta_{j}\right\rangle & =\int_{\mathbb{R}^{2}}\left[\prod_{j=0}^{1} \alpha_{j, \sigma}\left(\lambda_{j}\right)\right] d \nu\left(\lambda_{0}, \lambda_{1}\right),
\end{aligned}
$$

${ }^{\ddagger}$ For $\xi, \eta \in \mathcal{H}$, the one-dimensional operator $\xi \otimes \eta$ is defined as follows

$$
\xi \otimes \eta(\zeta)=\xi\langle\zeta, \eta\rangle \quad \zeta \in \mathcal{H}
$$


where $d \nu$ is the complex-valued product measure on $\mathbb{R}^{2}$

$$
d \nu=\prod_{j=0}^{1}\left\langle d E_{j} \xi_{j}, \eta_{j}\right\rangle
$$

Integrating (3.6) over $(\Sigma, d \mu)$, we now see that

$$
\operatorname{Tr}\left(x_{0} T\left(x_{1}\right)\right)=\int_{\mathbb{R}^{2}} \phi d \nu
$$

where

$$
\phi\left(\lambda_{0}, \lambda_{1}\right)=\int_{\Sigma}\left[\prod_{j=0}^{1} \alpha_{j, \sigma}\left(\lambda_{j}\right)\right] d \mu(\sigma)
$$

On the other hand, the assumption of the proof implies that $\phi=0$ and therefore the right hand side of (3) vanishes.

The above shows that (3.5) holds for one-dimensional operators $x_{j}, j=0,1$ and therefore it holds also for finite linear combination of such operators. It can be seen that the dual to the mapping $x_{1} \mapsto T\left(x_{1}\right)$ defined in (3.4) acts invariantly on the trace class $\mathfrak{S}^{1}$. In other words, the mapping (3.4) is ultra-weakly continuous. Since finite linear combinations of one-dimensional operators are ultra-weakly dense in $B(\mathcal{H})$, using (3.5) and the fact that the mapping

$$
\left(x_{0}, x_{1}\right) \mapsto \operatorname{Tr}\left(x_{0} T\left(x_{1}\right)\right)
$$

is bi-continuous with respect to the ultra-weak topology it now follows that (3.5) holds for any $x_{j} \in$ $B(\mathcal{H}), j=0,1$.

(ii) The estimate is rather straightforward. Let $\phi \in \mathfrak{A}$. Fix $\epsilon>0$ and a representation (3.1) such that

$$
\int_{\Sigma}\left[\prod_{j=0}^{1}\left\|\alpha_{j, \sigma}\right\|_{\infty}\right] d|\mu|(\sigma)<\|\phi\|_{\mathfrak{A}}+\epsilon
$$

Now, it is sufficient to note that, for every $\sigma \in \Sigma$, we have

$$
\left\|A_{0, \sigma} x_{1} A_{1, \sigma}\right\| \leq\left\|A_{0, \sigma}\right\|\left\|x_{1}\right\|\left\|A_{1, \sigma}\right\| \leq\left\|x_{1}\right\|\left[\prod_{j=0}^{1}\left\|\alpha_{j, \sigma}\right\|_{\infty}\right], \quad x_{1} \in B(\mathcal{H}) .
$$

Using the triangle inequality and the definition of $T_{\phi}$ we see

$$
\begin{aligned}
\left\|T_{\phi}\left(x_{1}\right)\right\| \leq \int_{\Sigma}\left\|A_{0, \sigma} x_{1} A_{1, \sigma}\right\| d|\mu| & (\sigma) \\
& \leq\left\|x_{1}\right\| \int_{\Sigma}\left[\prod_{j=0}^{1}\left\|\alpha_{j, \sigma}\right\|_{\infty}\right] d|\mu|(\sigma)<\left(\|\phi\|_{\mathfrak{A}}+\epsilon\right)\left\|x_{1}\right\| .
\end{aligned}
$$


Since the latter estimate is true for every $\epsilon>0$, we arrive at

$$
\left\|T_{\phi}\left(x_{1}\right)\right\| \leq\|\phi\|_{\mathfrak{A}}\left\|x_{1}\right\|, x_{1} \in B(\mathcal{H})
$$

Taking the supremum over all $x_{1} \in B(\mathcal{H})$ such that $\left\|x_{1}\right\| \leq 1$, the assertion (ii) follows.

The estimate in Lemma 1(ii) can easily be extended to noncommutative $L^{p}$-spaces. We fix a semifinite von Neumann algebra $\mathcal{M} \subseteq B(\mathcal{H})$ with normal semifinite faithful trace $\tau$. Let $L^{p}=$ $L^{p}(\mathcal{M}, \tau), 1 \leq p \leq \infty$ be the corresponding noncommutative $L^{p}$-space.

Lemma 2. Let $\phi \in \mathfrak{A}$. If the spectral measure $d E_{j}, j=0,1$ takes values in the algebra $\mathcal{M}$, then the operator $T_{\phi}$ acts invariantly on $\mathcal{M}$. Furthermore, if $1 \leq p \leq \infty$, then the operator $T_{\phi}$ admits a unique bounded extension to $L^{p}$. The latter extension is a bounded linear operator and

$$
\left\|T_{\phi}\right\|_{L^{p} \rightarrow L^{p}} \leq\|\phi\|_{\mathfrak{A}} .
$$

In particular,

$$
T_{\phi}(x) \prec \prec\|\phi\|_{\mathfrak{A}} x, \quad x \in \mathcal{M}+L^{1} .
$$

Proof. The invariance of $T_{\phi}$ on $\mathcal{M}$ is trivial.

The rest of the proof is basically a repetition of that of Lemma 1. We first consider the restriction of $T_{\phi}$ to $L^{p} \cap \mathcal{M}$. As in the proof of Lemma 1(ii) we see that

$$
\left\|T_{\phi}\left(x_{1}\right)\right\|_{p} \leq\|\phi\|_{\mathfrak{A}}\left\|x_{1}\right\|_{p}
$$

where $x_{1} \in L^{p} \cap \mathcal{M}$. The latter clearly implies that the operator $T_{\phi}$ restricted onto $L^{p} \cap \mathcal{M}$ is bounded. Thus, we only need to note that every bounded operator on $L^{p} \cap \mathcal{M}$ admits a unique extension to $L^{p}$ because the subspace $L^{p} \cap \mathcal{M}$ is norm dense in $L^{p}, p<\infty$.

Lemma 3. (i) The space $\mathfrak{A}$ is a Banach algebra.

(ii) The mapping $\phi \mapsto T_{\phi}$ is a continuous homomorphism of algebra $\mathfrak{A}$ into $B\left(L^{p}\right), 1 \leq p \leq \infty$.

(iii) If $f$ is a bounded Borel function and

$$
\phi_{0}\left(\lambda_{0}, \lambda_{1}\right)=f\left(\lambda_{0}\right) \text { and } \phi_{1}\left(\lambda_{0}, \lambda_{1}\right)=f\left(\lambda_{1}\right),
$$

then

$$
T_{\phi_{0}}(x)=F_{0} \cdot x \text { and } T_{\phi_{1}}(x)=x \cdot F_{1}
$$

where

$$
F_{j}=\int_{\mathbb{R}} f(\lambda) d E_{j}(\lambda)
$$

and $d E_{j}, j=0,1$ are the spectral measures underlying the mapping $\phi \mapsto T_{\phi}$. 
Proof. (i) The set $\mathfrak{A}$ is a linear space. In order to show that $\mathfrak{A}$ is a Banach algebra, we have to see that it is closed under the multiplication and that it is continuous with respect to the norm of $\mathfrak{A}$. The argument is standard. Let $\phi_{k} \in \mathfrak{A}, k=0,1$ and let $\epsilon>0$. Fix representations (3.1) for $\phi_{k}$, i.e., fix finite measure spaces $\left(\Sigma_{k}, d \mu_{k}\right), k=0,1$, and bounded Borel functions

$$
\alpha_{j, \sigma}^{(k)}: \mathbb{R} \mapsto \mathbb{C}, \quad \sigma \in \Sigma_{k} j=0,1, \quad k=0,1
$$

such that

$$
\phi_{k}\left(\lambda_{0}, \lambda_{1}\right)=\int_{\Sigma_{k}}\left[\prod_{j=0}^{1} \alpha_{j, \sigma}^{(k)}\left(\lambda_{j}\right)\right] d \mu_{k}(\sigma)
$$

and

$$
\int_{\Sigma_{k}}\left[\prod_{j=0}^{1}\left\|\alpha_{j, \sigma}^{(k)}\right\|_{\infty}\right] d\left|\mu_{k}\right|(\sigma)<\left\|\phi_{k}\right\|_{\mathfrak{A}}+\epsilon, \quad k=0,1 .
$$

Now, in order to show that $\phi=\phi_{0} \phi_{1}$ also belongs to $\mathfrak{A}$, we have to present a representation (3.1) for the function $\phi$. To this end, we choose the measure space $\left(\Sigma_{0} \times \Sigma_{1}, d \mu_{0} \times d \mu_{1}\right)$ and the bounded Borel function

$$
\alpha_{j, \sigma_{0}, \sigma_{1}}=\alpha_{j, \sigma_{0}}^{(0)} \alpha_{j, \sigma_{1}}^{(1)}, \quad \sigma_{k} \in \Sigma_{k}, \quad k=0,1, \quad j=0,1 .
$$

The fact that $\phi \in \mathfrak{A}$ and continuity of multiplication with respect to the norm of $\mathfrak{A}$ now follows from

$$
\begin{array}{r}
\left\|\phi_{0} \phi_{1}\right\|_{\mathfrak{A}}=\|\phi\|_{\mathfrak{A}} \\
\leq \int_{\Sigma_{1}} \int_{\Sigma_{0}} \prod_{j, k=0}^{1}\left\|\alpha_{j, \sigma_{k}}^{(k)}\right\|_{\infty} d\left|\mu_{0}\right|\left(\sigma_{0}\right) d\left|\mu_{1}\right|\left(\sigma_{1}\right) \\
\quad \leq\left(\left\|\phi_{0}\right\|_{\mathfrak{A}}+\epsilon\right)\left(\left\|\phi_{1}\right\|_{\mathfrak{A}}+\epsilon\right) .
\end{array}
$$

Part (i) is proved.

(ii) We continue with the above notation. In order to show that the mapping $\phi \mapsto T_{\phi}$ is a homomorphism, let us fix again $\phi_{k} \in \mathfrak{A}, k=0,1$ and the bounded Borel functions (3.7) such that (3.8) holds. Setting

$$
A_{j, \sigma_{k}}^{(k)}=\int_{\mathbb{R}} \alpha_{j, \sigma_{k}}^{(k)}(\lambda) d E_{j}(\lambda), \quad \sigma_{k} \in \Sigma_{k}, \quad j=0,1, \quad k=0,1,
$$

observing that the spectral theorem guarantees

$$
A_{j, \sigma_{0}, \sigma_{1}}=\int_{\mathbb{R}} \alpha_{j, \sigma_{0}, \sigma_{1}}(\lambda) d E_{j}(\lambda)=A_{j, \sigma_{0}}^{(0)} A_{j, \sigma_{1}}^{(1)}, \quad j=0,1,
$$


and refering to the definition of the operators $T_{\phi_{k}}, k=0,1$ and $T_{\phi}=T_{\phi_{0} \phi_{1}}$ (see (3.2)), we obtain

$$
\begin{aligned}
T_{\phi_{0} \phi_{1}}(x)=T_{\phi}(x) & \\
& =\int_{\Sigma \times \Sigma_{1}} A_{0, \sigma_{0}}^{(0)} A_{0, \sigma_{1}}^{(1)} x A_{1, \sigma_{1}}^{(1)} A_{1, \sigma_{0}}^{(0)} d\left(\mu_{0} \times \mu_{1}\right)\left(\sigma_{0}, \sigma_{1}\right) \\
& =\int_{\Sigma_{0}} A_{0, \sigma_{0}}^{(0)}\left[\int_{\Sigma_{1}} A_{0, \sigma_{1}}^{(1)} x A_{1, \sigma_{1}}^{(1)} d \mu_{1}\left(\sigma_{1}\right)\right] A_{1, \sigma_{0}}^{(0)} d \mu_{0}\left(\sigma_{0}\right)
\end{aligned}
$$$$
=T_{\phi_{0}}\left(T_{\phi_{1}}(x)\right)
$$

In other words,

$$
T_{\phi_{0} \phi_{1}}=T_{\phi_{0}} \cdot T_{\phi_{1}}
$$

i.e., the mapping $\phi \mapsto T_{\phi}$ is a (continuous) homomorphism. Part (ii) is proved.

(iii) It is sufficient to observe in this part that the functions $\phi_{j}, j=0,1$ are both belong to $\mathfrak{A}$. The assertion of part (iii) immediately follows from the definition of the operator $T_{\phi}$ (see (3.2)) and Lemma 1(i).

Now we prove the following perturbation lemma which will be the main tool in the following section.

Lemma 4. Let $f, \alpha_{j}$ and $\beta_{j}, j=0,1$ be Borel functions which are bounded on bounded subsets of $\mathbb{R}$ and let $H_{j} \in \mathcal{M}$ be self-adjoint, $j=0,1$. Let the function

$$
\phi\left(\lambda_{0}, \lambda_{1}\right):= \begin{cases}\frac{\beta_{0}\left(\lambda_{0}\right)}{\alpha_{0}\left(\lambda_{0}\right)} \cdot \frac{f\left(\lambda_{0}\right)-f\left(\lambda_{1}\right)}{\lambda_{0}-\lambda_{1}} \cdot \frac{\beta_{1}\left(\lambda_{1}\right)}{\alpha_{1}\left(\lambda_{1}\right)}, & \text { if }\left(\lambda_{0}, \lambda_{1}\right) \in \operatorname{Sp}\left(H_{0}\right) \times \operatorname{Sp}\left(H_{1}\right) ; \\ 0, & \text { otherwise }\end{cases}
$$

belong to $\mathfrak{A}$, where $\operatorname{Sp}\left(H_{j}\right)$ is the spectrum of the operator $H_{j}, j=0,1$. If $x \in \mathcal{M}$ and if $d E_{j}$ is the spectral measure of $H_{j}, j=0,1$, then

$$
B_{0}\left(F_{0} x-x F_{1}\right) B_{1}=T_{\phi}\left(A_{0}\left(H_{0} x-x H_{1}\right) A_{1}\right),
$$

where

$$
A_{j}=\alpha_{j}\left(H_{j}\right), \quad B_{j}=\beta_{j}\left(H_{j}\right), \quad F_{j}=f\left(H_{j}\right), \quad j=0,1 .
$$

In particular,

$$
\left\|B_{0}\left(F_{0} x-x F_{1}\right) B_{1}\right\|_{p} \leq\|\phi\|_{\mathfrak{A}}\left\|A_{0}\left(H_{0} x-x H_{1}\right) A_{1}\right\|_{p}, \quad 1 \leq p \leq \infty .
$$

Proof. Clearly (3.10) follows from (3.9) and Lemma 2. Thus, we need only to show (3.9). To this end, let

$$
\gamma_{j}\left(\lambda_{0}, \lambda_{1}\right)=\lambda_{j} \alpha_{0}\left(\lambda_{0}\right) \alpha_{1}\left(\lambda_{1}\right) \text { and } \delta_{j}\left(\lambda_{0}, \lambda_{1}\right)=f\left(\lambda_{j}\right) \beta_{0}\left(\lambda_{0}\right) \beta_{1}\left(\lambda_{1}\right), \quad j=0,1,
$$


where $\left(\lambda_{0}, \lambda_{1}\right) \in \operatorname{Sp}\left(H_{0}\right) \times \operatorname{Sp}\left(H_{1}\right)$. Note that the functions $\gamma_{j}$ and $\delta_{j}$ have trivial representations of the form (3.1) and therefore belong to $\mathfrak{A}$. Moreover, according to Lemma 3

$$
T_{\gamma_{0}}(x)=A_{0} H_{0} x A_{1}, T_{\gamma_{1}}(x)=A_{0} x H_{1} A_{1} \text { and } T_{\delta_{0}}(x)=B_{0} F_{0} x B_{1}, T_{\delta_{1}}(x)=B_{0} x F_{1} B_{1} .
$$

Finally, using again the fact that $\phi \mapsto T_{\phi}$ is a homomorphism, we see

$$
\begin{aligned}
T_{\phi}\left(A_{0}\left(H_{0} x-x H_{1}\right) A_{1}\right)=T_{\phi}\left(T_{\gamma_{0}}(x)\right. & \left.-T_{\gamma_{1}}(x)\right) \\
& =T_{\phi\left(\gamma_{0}-\gamma_{1}\right)}(x)=T_{\delta_{0}-\delta_{1}}(x)=B_{0}\left(F_{0} x-x F_{1}\right) B_{1} .
\end{aligned}
$$

Remark 5. A quick inspection of the proof above shows that the following relation

$$
B_{0}\left(F_{0} x+x F_{1}\right) B_{1}=T_{\phi}\left(A_{0}\left(H_{0} x+x H_{1}\right) A_{1}\right),
$$

where

$$
\phi\left(\lambda_{0}, \lambda_{1}\right)= \begin{cases}\frac{\beta_{0}\left(\lambda_{0}\right)}{\alpha_{0}\left(\lambda_{0}\right)} \cdot \frac{f\left(\lambda_{0}\right)+f\left(\lambda_{1}\right)}{\lambda_{0}+\lambda_{1}} \cdot \frac{\beta_{1}\left(\lambda_{1}\right)}{\alpha_{1}\left(\lambda_{1}\right)}, & \text { if }\left(\lambda_{0}, \lambda_{1}\right) \in \operatorname{Sp}\left(H_{0}\right) \times \operatorname{Sp}\left(H_{1}\right) ; \\ 0, & \text { otherwise }\end{cases}
$$

also holds, provided $\phi \in \mathfrak{A}$. Thus, we also have

$$
\left\|B_{0}\left(F_{0} x+x F_{1}\right) B_{1}\right\|_{p} \leq\|\phi\|_{\mathfrak{A}}\left\|A_{0}\left(H_{0} x+x H_{1}\right) A_{1}\right\|_{p}, \quad 1 \leq p \leq \infty .
$$

Lemma 4 and Remark 5 suggest the following simple corollary.

Lemma 6. If $f$ is a $C^{2}$-function, then, for every self-adjoint $H_{j} \in \mathcal{M}, j=0,1$,

$$
\left\|F_{0}-F_{1}\right\|_{p} \leq c\left\|H_{0}-H_{1}\right\|_{p} \max _{m=0,1,2}\left\|f^{(m)}\right\|_{\infty}, \quad 1 \leq p \leq \infty,
$$

where $c>0$ is a universal constant and where $F_{j}=f\left(H_{j}\right), j=0,1$.

Proof. The proof uses Theorem 4 of [24]. Let $\alpha_{j}=\beta_{j}=1$ and the function $\phi$ of Lemma 4 be given by

$$
\phi\left(\lambda_{0}, \lambda_{1}\right)=\frac{f\left(\lambda_{0}\right)-f\left(\lambda_{1}\right)}{\lambda_{0}-\lambda_{1}} .
$$

It follows from [24, Theorem 4] that $\phi \in \mathfrak{A}$ and

$$
\|\phi\|_{\mathfrak{A}} \leq c \max _{m=0,1,2}\left\|f^{(m)}\right\|_{\infty} .
$$

Now the required estimate follows from Lemma 4 since

$$
F_{0}-F_{1}=T_{\phi}\left(H_{0}-H_{1}\right) \text {. }
$$


Let us now consider the subclass $\mathfrak{C}$ within the algebra $\mathfrak{A}$ which is defined as follows. A function $\phi \in \mathfrak{A}$ belongs to $\mathfrak{C}$ if and only if there is a representation (3.1) with the additional property that there is a increasing sequence of measurable subsets $S_{k} \subseteq \Sigma, k=1,2, \ldots$ such that

$$
\Sigma=\bigcup_{k=1}^{\infty} S_{k}
$$

and such that the family of functions $\left\{\alpha_{j, \sigma}\right\}_{\sigma \in S_{k}}$ is uniformly continuous for every $j=0,1$ and $k=$ $1,2, \ldots$

Lemma 7. Let $\phi \in \mathfrak{C}$. Let $1 \leq p \leq \infty$ and let $\left\{H_{t}\right\}_{t \in \mathbb{R}} \subseteq \mathcal{M}$,

$$
H_{t}=\int_{\mathbb{R}} \lambda d E_{t}(\lambda)
$$

be a family of self-adjoint operators such that

$$
\lim _{t \rightarrow 0}\left\|H_{t}-H_{0}\right\|=0 .
$$

If $T_{\phi, t}$ is the double operator integral associated with $\phi$ and the measures $d E_{t}$ and $d E_{0}$, then

$$
\lim _{t \rightarrow 0}\left\|T_{\phi, t}(x)-T_{\phi, 0}(x)\right\|_{p}=0, \quad x \in L^{p} .
$$

Proof. The proof is rather standard and uses several stages of approximation. Let us fix a measure space $(\Sigma, d \mu)$ and bounded Borel functions $\alpha_{j, \sigma}, j=0,1, \sigma \in \Sigma$ such that (3.1) holds. Moreover, let $S_{k} \subseteq \Sigma, k=1,2, \ldots$ be an increasing sequence of measurable subsets such that

$$
\Sigma=\bigcup_{k=1}^{\infty} S_{k}
$$

and the family $\left\{\alpha_{j, \sigma}\right\}_{\sigma \in S_{k}}$ is uniformly continuous for every $j=0,1$ and $k=1,2, \ldots$

Fix $\epsilon>0$. At first, we shall show that there is a function $\phi_{\epsilon} \in \mathfrak{C}$ such that

$$
\left\|\phi-\phi_{\epsilon}\right\|_{\mathfrak{A}}<\epsilon
$$

and such that the representatives $\alpha_{j, \sigma, \epsilon}, j=0,1, \sigma \in \Sigma$ from (3.1) for $\phi_{\epsilon}$ have the additional property that the family

$$
\left\{\alpha_{j, \sigma, \epsilon}^{(m)}\right\}_{\sigma \in S_{k}}
$$

is uniformly continuous for every $j=0,1$, every $k=1,2, \ldots$ and every $m=0,1, \ldots$, where $\alpha_{j, \sigma, \epsilon}^{(m)}$ is the $m$-th derivative of $\alpha_{j, \sigma, \epsilon}$. To this end, we shall employ the standard smoothing technique of harmonic analysis. Fix $\epsilon>0$ and let $k_{\epsilon} \in \mathbb{N}$ be an integer such that

$$
\int_{\Sigma \backslash S_{k_{\epsilon}}}\left[\prod_{j=0}^{1}\left\|\alpha_{j, \sigma}\right\|_{\infty}\right] d|\mu|(\sigma)<\frac{\epsilon}{3} .
$$


Now, we set

$$
\alpha_{j, \sigma, \epsilon}=\left\{\begin{array}{ll}
\alpha_{j, \sigma} * P_{y_{\epsilon}}, & \text { if } \sigma \in S_{k_{\epsilon}} \\
0, & \text { otherwise }
\end{array},\right.
$$

where $P_{y_{\epsilon}}$ is the Poisson kernel

$$
P_{y_{\epsilon}}(x)=\frac{1}{\pi} \frac{y_{\epsilon}}{x^{2}+y_{\epsilon}^{2}}, \quad x \in \mathbb{R}, y_{\epsilon} \geq 0
$$

with $y_{\epsilon}$ so large that

$$
\left\|\alpha_{j, \sigma} * P_{\epsilon}-\alpha_{j, \sigma}\right\|_{\infty}<\frac{\epsilon}{K_{\epsilon}}, j=0,1
$$

and where

$$
K_{\epsilon}=3|\mu|\left(S_{k_{\epsilon}}\right) \sup _{\sigma \in S_{k_{\epsilon}}} \max _{j=0,1}\left\|\alpha_{j, \sigma}\right\|_{\infty} .
$$

Note that the constant $K_{\epsilon}$ is always finite and $y_{\epsilon}>0$ always exists, both due to the fact that the families of functions $\left\{\alpha_{j, \sigma}\right\}_{\sigma \in S_{k_{\epsilon}}}, j=0,1$ are uniformly continuous and $\left\{P_{y}\right\}_{y>0}$ is an approximation identity. We now observe that if

$$
\phi_{\epsilon}\left(\lambda_{0}, \lambda_{1}\right)=\int_{\Sigma}\left[\prod_{j=0}^{1} \alpha_{j, \sigma, \epsilon}\left(\lambda_{j}\right)\right] d \mu(\sigma)=\int_{S_{k_{\epsilon}}}\left[\prod_{j=0}^{1} \alpha_{j, \sigma, \epsilon}\left(\lambda_{j}\right)\right] d \mu(\sigma),
$$

then

$$
\begin{aligned}
\phi\left(\lambda_{0}, \lambda_{1}\right)-\phi_{\epsilon}\left(\lambda_{0}, \lambda_{1}\right) & \\
& =\int_{S_{k_{\epsilon}}} \alpha_{0, \sigma, \epsilon}\left(\lambda_{0}\right)\left[\alpha_{1, \sigma, \epsilon}\left(\lambda_{1}\right)-\alpha_{1, \sigma}\left(\lambda_{1}\right)\right] d \mu(\sigma) \\
& +\int_{S_{k_{\epsilon}}}\left[\alpha_{0, \sigma, \epsilon}\left(\lambda_{0}\right)-\alpha_{0, \sigma}\left(\lambda_{0}\right)\right] \alpha_{1, \sigma}\left(\lambda_{1}\right) d \mu(\sigma) \\
& +\int_{\Sigma \backslash S_{k_{\epsilon}}}\left[\prod_{j=0}^{1} \alpha_{j, \sigma}\left(\lambda_{j}\right)\right] d \mu(\sigma) .
\end{aligned}
$$

Every term above is controlled by $\frac{\epsilon}{3}$ due to the choice of $\alpha_{j, \sigma, \epsilon}$ and $S_{k_{\epsilon}}$ and therefore we arrive at

$$
\left\|\phi-\phi_{\epsilon}\right\|_{\mathfrak{A}} \leq \epsilon
$$

Furthermore, again recalling that the family $\left\{\alpha_{j, \sigma}\right\}_{\sigma \in S_{k_{\epsilon}}}, j=0,1$ is uniformly continuous, it follows from the properties of Poisson kernel that the family $\left\{\alpha_{j, \sigma, \epsilon}\right\}_{\sigma \in S_{k}}$ is uniformly $C^{\infty}$, i.e., the derived families

$$
\left\{\alpha_{j, \sigma, \epsilon}^{(m)}\right\}_{\sigma \in S_{k \epsilon}}
$$


are uniformly continuously for every $m=0,1, \ldots$ This completes the first stage of approximation.

At the second stage, let us show that if $T_{\phi_{\epsilon}, t}$ is the double operator integral associated with $\phi_{\epsilon}$ and the spectral measures $d E_{t}$ and $d E_{0}$, then there is $\delta>0$ such that

$$
\left\|T_{\phi_{\epsilon}, t}(x)-T_{\phi_{\epsilon}, 0}(x)\right\|_{p}<\epsilon\|x\|_{p}, \text { if }|t|<\delta .
$$

In order to show (3.11), let

$$
A_{j, \sigma, \epsilon, t}=\alpha_{j, \sigma, \epsilon}\left(H_{t}\right)=\int_{\mathbb{R}} \alpha_{j, \sigma, \epsilon}(\lambda) d E_{t}(\lambda)
$$

By Lemma 6, we have

$$
\left\|A_{j, \sigma, \epsilon, t}-A_{j, \sigma, \epsilon, 0}\right\| \leq K_{\epsilon}^{\prime}\left\|H_{t}-H_{0}\right\|,
$$

where

$$
K_{\epsilon}^{\prime}=\sup _{\sigma \in S_{k_{\epsilon}}} \max _{j=0,1} \max _{m=0,1,2}\left\|\alpha_{j, \sigma, \epsilon}^{(m)}\right\|_{\infty} .
$$

By the assumption

$$
\lim _{t \rightarrow 0}\left\|H_{t}-H_{0}\right\|=0,
$$

and so there is $\delta>0$ such that

$$
\left\|A_{j, \sigma, \epsilon, t}-A_{j, \sigma, \epsilon, 0}\right\|<\frac{\epsilon}{K_{\epsilon}^{\prime \prime}}, \text { if }|t|<\delta,
$$

where

$$
K_{\epsilon}^{\prime \prime}=\sup _{\sigma \in S_{k_{\epsilon}}}\left\|\alpha_{1, \sigma, \epsilon}\right\|_{\infty}
$$

Finally, since

$$
T_{\phi_{\epsilon}, t}(x)-T_{\phi_{\epsilon}, 0}(x)=\int_{S_{k_{\epsilon}}}\left[A_{0, \sigma, \epsilon, t}-A_{0, \sigma, \epsilon, 0}\right] x A_{1, \sigma, \epsilon, 0} d \mu(\sigma),
$$

we estimate

$$
\left\|T_{\phi_{\epsilon}, t}(x)-T_{\phi_{0}, 0}(x)\right\|_{p} \leq \epsilon\|x\|_{p} .
$$

This finishes the second stage of the proof.

Now, at the final stage, it is left to observe that for every $|t|<\delta$,

$$
\begin{aligned}
\left\|T_{\phi, t}(x)-T_{\phi, 0}(x)\right\|_{p} \leq \| T_{\phi, t}(x) & -T_{\phi_{\epsilon}, t}(x) \|_{p} \\
& +\left\|T_{\phi_{\epsilon}, t}(x)-T_{\phi_{\epsilon}, 0}(x)\right\|_{p}+\left\|T_{\phi_{\epsilon}, 0}(x)-T_{\phi, 0}(x)\right\|_{p} \leq 3 \epsilon\|x\|_{p} .
\end{aligned}
$$

Here every term is controlled by $\epsilon\|x\|_{p}$ : the first and the third due to the fact that $\left\|\phi-\phi_{\epsilon}\right\|_{\mathfrak{A}}<\epsilon$ and the second term due to the choice of $|t|<\delta$ (see (3.11)). The lemma is completely proved. 
The combination of Lemma 4 and 7 implies the following corollary.

Lemma 8. Let $1 \leq p \leq \infty$ and let $\left\{H_{t}\right\}_{t \in \mathbb{R}} \subseteq \mathcal{M}$ be a self-adjoint family of operators such that

$$
\lim _{t \rightarrow 0}\left\|H_{t}-H_{0}\right\|=0 .
$$

Let $\alpha_{j}, \beta_{j}$ and $\phi$ be as in Lemma 4 and let

$$
A_{0}=\alpha_{0}\left(H_{0}\right), A_{t}=\alpha_{1}\left(H_{t}\right) \text { and } B_{0}=\beta_{0}\left(H_{0}\right), B_{t}=\beta_{1}\left(H_{t}\right)
$$

If $\phi \in \mathfrak{C}$ and if

$$
D_{0}=\lim _{t \rightarrow 0} A_{t} \frac{H_{t}-H_{0}}{t} A_{0}
$$

exists in norm of $L^{p}$, then the limit

$$
G_{0}=\lim _{t \rightarrow 0} B_{t} \frac{F_{t}-F_{0}}{t} B_{0}
$$

exists in norm of $L^{p}$ also and

$$
G_{0}=T_{\phi}\left(D_{0}\right)
$$

where $T_{\phi}$ is the double operator integral associated with $\phi$ and the spectral measures $d E_{j}, j=0,1$, where $d E_{0}=d E_{1}$ is the spectral measure of $H_{0}$.

Proof. The proof is immediate. If $T_{\phi, t}$ is the double operator integral associated with $\phi$ and the spectral measures of $H_{t}$ and $H_{0}$ respectively and if

$$
D_{t}=A_{t} \frac{H_{t}-H_{0}}{t} A_{0} \text { and } G_{t}=B_{t} \frac{F_{t}-F_{0}}{t} B_{0}
$$

then by Lemma 4 ,

$$
G_{t}=T_{\phi, t}\left(D_{t}\right)
$$

Consequently, by setting $G_{0}=T_{\phi, 0}\left(D_{0}\right)$,

$$
\lim _{t \rightarrow 0}\left\|G_{t}-G_{0}\right\|_{p}=\lim _{t \rightarrow 0}\left\|T_{\phi, t}\left(D_{t}-D_{0}\right)\right\|+\left\|\left[T_{\phi, t}-T_{\phi}\right]\left(D_{0}\right)\right\|_{p} .
$$

The first limit vanishes due to the assumption that $\lim _{t \rightarrow 0} D_{t}=D_{0}$ and the second vanishes due to Lemma 7. 


\section{Operator inequalities}

Here we present a unified method of proving a number of operator inequalities using double operator integrals and the perturbation Lemma 4. The idea of this approach is taken from our paper [24]. The major technical ingredient is given in the following theorem.

Theorem 9. Let $g: \mathbb{R} \mapsto \mathbb{C}$ be an $L^{2}$-function with $L^{2}$-derivative $g^{\prime}$, i.e.,

$$
\|g\|_{2}<+\infty \text { and }\left\|g^{\prime}\right\|_{2}<+\infty
$$

If

$$
\phi\left(\lambda_{0}, \lambda_{1}\right)=g\left(\log \frac{\lambda_{0}}{\lambda_{1}}\right), \lambda_{j}>0, j=0,1,
$$

then $\phi \in \mathfrak{C}$ and

$$
\|\phi\|_{\mathfrak{A}} \leq \sqrt{2}\left(\|g\|_{2}+\left\|g^{\prime}\right\|_{2}\right)
$$

Proof. It follows from [24, Lemma 7] that the function $g$ has integrable Fourier transform $\hat{g}$ and

$$
\|\hat{g}\|_{1} \leq \sqrt{2}\left(\|g\|_{2}+\left\|g^{\prime}\right\|_{2}\right)
$$

On the other hand, if $g$ has integrable Fourier transform, then,

$$
g(t)=\int_{\mathbb{R}} \hat{g}(s) e^{i t s} d s
$$

Setting $t=\log \frac{\lambda_{0}}{\lambda_{1}}$, we have

$$
\phi\left(\lambda_{0}, \lambda_{1}\right)=g\left(\log \frac{\lambda_{0}}{\lambda_{1}}\right)=\int_{\mathbb{R}} \hat{g}(s) \lambda_{0}^{i s} \lambda_{1}^{-i s} d s .
$$

To show that $\phi$ belongs to $\mathfrak{A}$, we have to present a measure space $(\Sigma, d \mu)$, with finite total variation of measure $\mu$ and bounded Borel functions $\alpha_{j, s}$ such that (3.1) holds. We choose $\Sigma=\mathbb{R}$ and $d \mu(\sigma)=\hat{g}(\sigma) d \sigma$ and

$$
\alpha_{0, \sigma}(\lambda)=\lambda^{i \sigma}, \quad \alpha_{1, \sigma}(\lambda)=\lambda^{-i \sigma}, \quad \sigma \in \Sigma
$$

and the representation (3.1) follows from (4.1). Furthermore, since $\left\|\alpha_{j, \sigma}\right\| \leq 1, j=0,1$, we have

$$
\|\phi\|_{\mathfrak{A}} \leq \int_{\Sigma} d|\mu|(\sigma)=\int_{\mathbb{R}}|\hat{g}(s)| d s=\|\hat{g}\|_{1} \leq \sqrt{2}\left(\|g\|_{2}+\left\|g^{\prime}\right\|_{2}\right) .
$$

Observe also that if $S_{k}$ is the increasing family of subintervals $[-k, k], k=1,2, \ldots$ then the family $\left\{\alpha_{j, \sigma}\right\}_{\sigma \in S_{k}}$ is uniformly continuous, $j=0,1, k=1,2, \ldots$ 
We shall also frequently use the following elementary lemma.

Lemma 10. The functions $g, g^{\prime}$ and $\tilde{g}, \tilde{g}^{\prime}$ are square summable, i.e., $g, g^{\prime}, \tilde{g}, \tilde{g}^{\prime} \in L^{2}(\mathbb{R})$, where

$$
g(t)=\frac{e^{\alpha_{0} t}-e^{-\alpha_{1} t}}{e^{\beta_{0} t}-e^{-\beta_{1} t}} \text { and } \tilde{g}(t)=\frac{e^{\alpha_{0} t}+e^{-\alpha_{1} t}}{e^{\beta_{0} t}+e^{-\beta_{1} t}}
$$

and where $0 \leq \operatorname{Re} \alpha_{j}<\operatorname{Re} \beta_{j}, j=0,1$.

The proof of the lemma is elementary and left to the reader.

We shall now prove a number of known inequalities using the techniques above. We would like to note that although our methods below do not always provide the best possible constants in estimates, they certainly have potential for further generalisations and extensions.

\section{The Heinz inequality.}

The origin of the following inequality can be found in [20]. For an elegant alternative proof, we refer to [21]. A detailed discussion of this inequality and its analogues is contained in [15].

Lemma 11. Let $A, B \in \mathcal{M}$ be positive and let $x \in \mathcal{M}$. For every $0 \leq \theta \leq 1$, there is a constant $c_{\theta}>0$ such that for every $1 \leq p \leq \infty$,

$$
\left\|A^{1-\theta} x B^{\theta}+A^{\theta} x B^{1-\theta}\right\|_{p} \leq c_{\theta}\|A x+x B\|_{p} .
$$

Proof. Note that the claim of the lemma is trivial if $\theta=0,1$. We shall assume that $0<\theta<1$.

Let us employ Remark 5. It shows that

$$
A^{1-\theta} x B^{\theta}+A^{\theta} x B^{1-\theta}=T_{\phi_{\theta}}(A x+x B)
$$

provided $\phi_{\theta}$ belongs to $\mathfrak{A}$, where the function $\phi_{\theta}$ is given by

$$
\phi_{\theta}\left(\lambda_{0}, \lambda_{1}\right)=\frac{\lambda_{0}^{1-\theta} \lambda_{1}^{\theta}+\lambda_{0}^{\theta} \lambda_{1}^{1-\theta}}{\lambda_{0}+\lambda_{1}}=g_{\theta}(t), \quad t=\log \frac{\lambda_{0}}{\lambda_{1}},
$$

and where

$$
g_{\theta}(t)=\frac{e^{\left(\frac{1}{2}-\theta\right) t}+e^{\left(\theta-\frac{1}{2}\right) t}}{e^{\frac{t}{2}}+e^{-\frac{t}{2}}} .
$$

On the other hand, the function $g_{\theta}$ is $L^{2}$ and its first derivative is also $L^{2}$ (see Lemma 10). Consequently, according to Theorem $9, \phi_{\theta}$ indeed belongs to $\mathfrak{A}$. Thus, the estimate of the lemma follows. 
Remark 12. A minor modification of the proof shows also that

$$
\left\|A^{1-\theta} x B^{\theta}-A^{\theta} x B^{1-\theta}\right\|_{p} \leq c_{\theta}\|A x-x B\|_{p}, \quad 1 \leq p \leq \infty
$$

Indeed, the estimate will follow if we replace the function $\phi_{\theta}$ with the function

$$
\tilde{\phi}_{\theta}\left(\lambda_{0}, \lambda_{1}\right)=\frac{\lambda_{0}^{1-\theta} \lambda_{1}^{\theta}-\lambda_{0}^{\theta} \lambda_{1}^{1-\theta}}{\lambda_{0}-\lambda_{1}}=\tilde{g}_{\theta}(t),
$$

where

$$
\tilde{g}(t)=\frac{e^{\left(\frac{1}{2}-\theta\right) t}-e^{\left(\theta-\frac{1}{2}\right) t}}{e^{\frac{t}{2}}-e^{-\frac{t}{2}}} .
$$

Since $\tilde{g}, \tilde{g}^{\prime} \in L^{2}$, the inequality follows from Theorem 9.

Remark 13. Since there is a linear operator

$$
T: \mathcal{M}+L^{1} \mapsto \mathcal{M}+L^{1}
$$

such that

$$
\left.T\right|_{\mathcal{M}} \in B(\mathcal{M}) \text { and }\left.T\right|_{L^{1}} \in B\left(L^{1}\right)
$$

and such that

$$
A^{1-\theta} x B^{\theta}+A^{\theta} x B^{1-\theta}=T(A x+x B),
$$

we also have the submajorisation

$$
A^{1-\theta} x B^{\theta}+A^{\theta} x B^{1-\theta} \prec \prec c_{\theta}(A x+x B)
$$

\section{The inequality of Connes and Moscovici.}

The following lemma was stated in [10]. Let $D$ be a self-adjoint operator on $\mathcal{H}$.

Lemma 14 (Lemma 8 of [10]). Let $\alpha \in[0,1]$ and $D_{\alpha}=D|D|^{-\alpha}$. If $p^{\prime}>p$, then $\left[D_{\alpha}, a\right] \in L^{\frac{p^{\prime}}{\alpha}}$ for every $a \in \mathcal{A}$ and

$$
\left\|\left[D_{\alpha}, a\right]\right\|_{\frac{p^{\prime}}{\alpha}} \leq c_{p, p^{\prime}, \alpha}\|[D, a]\|_{\infty}\left(1+\left\|D^{-1}\right\|_{p}^{2}\right)
$$

where $c_{p, p^{\prime}, \alpha}>0$ is a numerical constant and $\mathcal{A}$ is the collection of operators such that $[D, a] \in$ $L^{\infty}$.

Using double operator integral techniques and ideas from [24, 9], the lemma above may be improved as follows. 
Lemma 15. Let $\alpha \in[0,1], D_{\alpha}=D|D|^{-\alpha}$. Suppose that $a \in \mathcal{A}$, and that $D \in \mathcal{M}$ is self-adjoint ${ }^{\S}$. For every $0 \leq \alpha \leq 1$, there is a constant $c_{\alpha}>0$ such that

$$
\left\|\left[D_{\alpha}, a\right]\right\|_{\frac{p}{\alpha}} \leq c_{\alpha}\|[D, a]\|_{\infty}\left\||D|^{-1}\right\|_{p}^{\alpha}, \quad \alpha \leq p \leq \infty
$$

Proof of Lemma 15. The claim of the lemma is trivial if $\alpha=0$. Assume that $\alpha>0$. We shall prove that

$$
\left\|\left[D_{\alpha}, a\right]\right\|_{q} \leq c_{\alpha}\left\||D|^{-\frac{\alpha}{2}}[D, a]|D|^{-\frac{\alpha}{2}}\right\|_{q}, \quad 1 \leq q \leq \infty .
$$

If we assume that (4.2) is proved, then, by taking $q=\frac{p}{\alpha}$ and using the Hölder inequality,

$$
\left\|\left[D_{\alpha}, a\right]\right\|_{\frac{p}{\alpha}} \leq c_{\alpha}\|[D, a]\|_{\infty}\left\||D|^{-\frac{\alpha}{2}}\right\|_{\frac{2 p}{\alpha}}^{2}=c_{\alpha}\|[D, a]\|_{\infty}\left\||D|^{-1}\right\|_{p}^{\alpha}
$$

and the claim of the lemma follows.

In order to prove (4.2), we use Lemma 4. If

$$
\phi\left(\lambda_{0}, \lambda_{1}\right)=\left|\lambda_{0}\right|^{\frac{\alpha}{2}} \frac{\left|\lambda_{0}\right|^{1-\alpha} \operatorname{sgn} \lambda_{0}-\left|\lambda_{1}\right|^{1-\alpha} \operatorname{sgn} \lambda_{1}}{\lambda_{0}-\lambda_{1}}\left|\lambda_{1}\right|^{\frac{\alpha}{2}} .
$$

and if $\phi \in \mathfrak{A}$, then

$$
\left[D_{\alpha}, a\right]=T_{\phi}\left(|D|^{-\frac{\alpha}{2}}[D, a]|D|^{-\frac{\alpha}{2}}\right)
$$

where $T_{\phi}$ is the double operator integral associated with the function. Thus, the estimate (4.2) will follow as soon as we prove that the function $\phi$ above belongs to $\mathfrak{A}$. We shall decompose the function $\phi$ into four parts

$$
\phi=\phi_{++}+\phi_{+-}+\phi_{-+}+\phi_{--},
$$

where

$$
\phi_{++}\left(\lambda_{0}, \lambda_{1}\right)=\chi_{\mathbb{R}^{+}}\left(\lambda_{0}\right) \phi\left(\lambda_{0}, \lambda_{1}\right) \chi_{\mathbb{R}^{+}}\left(\lambda_{1}\right)
$$

and the other three parts are the restrictions of $\phi$ to the other three quadrants of the plane $\mathbb{R}^{2}$.

We shall consider the parts $\phi_{++}$and $\phi_{+-}$. The argument for the other two parts can be reduced to these two.

(i) If we set $t=\log \frac{\lambda_{0}}{\lambda_{1}}$, then

$$
\phi_{++}\left(\lambda_{0}, \lambda_{1}\right)=\lambda_{0}^{\frac{\alpha}{2}} \lambda_{1}^{\frac{\alpha}{2}} \frac{\lambda_{0}^{1-\alpha}-\lambda_{1}^{1-\alpha}}{\lambda_{0}-\lambda_{1}}=g_{1}(t)
$$

where

$$
g_{1}(t)=\frac{e^{(1-\alpha) \frac{t}{2}}-e^{(\alpha-1) \frac{t}{2}}}{e^{\frac{t}{2}}-e^{-\frac{t}{2}}}
$$

\footnotetext{
$\S$ We require $D$ to be bounded only for the simplicity of the present exposition. The result and the proof remain correct even if $D$ is unbounded though the argument would become more technical.
} 
Consequently, since $\alpha>0$, the function $g_{1}$ belongs to $L^{2}$ together with its first derivative $g_{1}^{\prime}$ (see Lemma 10). Thus, according to Theorem $9, \phi_{++}$belongs to $\mathfrak{A}$.

(ii) $\lambda_{0}>0$ and $\lambda_{1}<0$. The argument is very similar to the above. If $t=\log \frac{\lambda_{0}}{\left|\lambda_{1}\right|}$, then

$$
\phi_{+-}\left(\lambda_{0}, \lambda_{1}\right)=\lambda_{0}^{\frac{\alpha}{2}}\left|\lambda_{1}\right|^{\frac{\alpha}{2}} \frac{\lambda_{0}^{1-\alpha}+\left|\lambda_{1}\right|^{1-\alpha}}{\lambda_{0}+\left|\lambda_{1}\right|}=g_{2}(t),
$$

where

$$
g_{2}(t)=\frac{e^{(1-\alpha) \frac{t}{2}}+e^{(\alpha-1) \frac{t}{2}}}{e^{\frac{t}{2}}+e^{-\frac{t}{2}}} .
$$

Here, due to the observation that $g_{2} \in L^{2}(\mathbb{R})$ and $g_{2}^{\prime} \in L^{2}(\mathbb{R})$ if $\alpha>0$, Lemma 10 and Theorem 9 yield that $\phi_{+-} \in \mathfrak{A}$.

\section{The inequality of Birman, Koplienko and Solomyak}

This inequality was initially proved in [3] with an alternative proof (applicable to a large class of operator-functions) given in [1]. Ando's proof was later extended to semifinite von Neumann algebras in [11]. Although our approach is similar to the original approach of [3], some important technical details are different.

Theorem 16. If $H_{j} \in \mathcal{M}, j=0,1$ are positive operators and $0 \leq \alpha \leq 1$, then

$$
\left\|H_{1}^{\alpha}-H_{0}^{\alpha}\right\|_{\frac{p}{\alpha}} \leq c_{\alpha}\left\|H_{1}-H_{0}\right\|_{p}^{\alpha}, \quad \alpha \leq p \leq \infty
$$

for some numerical constant $c_{\alpha}>0$.

Proof. The special case $\alpha=1$ is trivial. We shall assume that $\alpha<1$. Furthermore, we may also assume the operators $H_{j}, j=0,1$ are invertible?

We first show that we may also assume that $H_{1}-H_{0}$ is positive. Indeed, assume that for the moment that the estimate (4.3) is true with $H_{1}-H_{0}>0$. Now, for arbitrary $H_{1}-H_{0}$, we introduce

$$
H_{+}=H_{0}+\left(H_{1}-H_{0}\right)^{+} \text {and } H_{-}=H_{0}-\left(H_{1}-H_{0}\right)^{-} .
$$

Observe that since the function $t \mapsto t^{\alpha}, 0 \leq \alpha \leq 1$ is operator-monotone,

$$
H_{-}^{\alpha}-H_{0}^{\alpha} \leq H_{1}^{\alpha}-H_{0}^{\alpha} \leq H_{+}^{\alpha}-H_{0}^{\alpha} \text {. }
$$

Because the right hand side is positive and the left hand side is negative and using [27], we have

$$
\left\|H_{1}^{\alpha}-H_{0}^{\alpha}\right\|_{\frac{p}{\alpha}} \leq 2 \max \left\{\left\|H_{0}^{\alpha}-H_{-}^{\alpha}\right\|_{\frac{p}{\alpha}},\left\|H_{+}^{\alpha}-H_{0}^{\alpha}\right\|_{\frac{p}{\alpha}}\right\} .
$$

` Otherwise, we replace $H_{j}$ with $H_{j}+\epsilon$ and then let $\epsilon \rightarrow 0$. 
On the other hand, we estimate the latter with (4.3) since the differences $H_{+}-H_{0}$ and $H_{0}-H_{-}$ are positive, i.e.,

$$
\left\|H_{+}^{\alpha}-H_{0}^{\alpha}\right\|_{\frac{p}{\alpha}} \leq c_{\alpha}\left\|H_{+}-H_{0}\right\|_{p}^{\alpha}=c_{\alpha}\left\|\left(H_{1}-H_{0}\right)^{+}\right\|_{p}^{\alpha} \leq c_{\alpha}\left\|H_{1}-H_{0}\right\|_{p}^{\alpha}
$$

and similarly for $\left\|H_{0}^{\alpha}-H_{-}^{\alpha}\right\|_{\frac{p}{\alpha}}$.

So, from now on, we assume that $H_{j}$ are invertible and $H_{1}-H_{0}$ is positive.

Let $\delta=\frac{1}{2}(1-\alpha)$ for brevity. Let $H_{t}=H_{0}+t\left(H_{1}-H_{0}\right)$ and let us show that

$$
D_{t}=\lim _{\Delta t \rightarrow 0} H_{t+\Delta t}^{-\delta} \frac{H_{t+\Delta t}-H_{t}}{\Delta t} H_{t}^{-\delta}=\left[\lim _{\Delta t \rightarrow 0} H_{t+\Delta t}^{-\delta}\right]\left(H_{1}-H_{0}\right) H_{t}^{-\delta}=H_{t}^{-\delta}\left(H_{1}-H_{0}\right) H_{t}^{-\delta}
$$

exists with respect to the norm of $L^{\frac{p}{\alpha}}$. Indeed, the second and the first factors are trivially bounded (since $H_{j} \in \mathcal{M}, j=0,1$ ) and it follows from Lemma 6 since the function $t \mapsto t^{-\delta}$ is $C^{2}$ on the spectrum of $H_{t}$ (recall that $H_{t} \geq H_{0}$ and therefore is invertible) and thus

$$
\left\|H_{t+\Delta t}^{-\delta}-H_{t}^{-\delta}\right\| \leq c_{\alpha} \Delta t\left\|H_{1}-H_{0}\right\|
$$

Consider now the function

$$
\phi_{\alpha}\left(\lambda_{0}, \lambda_{1}\right)= \begin{cases}\lambda_{0}^{\delta} \lambda_{1}^{\delta} \frac{\lambda_{0}^{\alpha}-\lambda_{1}^{\alpha}}{\lambda_{0}-\lambda_{1}}, & \text { if } \lambda_{j}>0, j=0,1 \\ 0, & \text { otherwise. }\end{cases}
$$

A moment's reflection shows that

$$
\phi_{\alpha}\left(\lambda_{0}, \lambda_{1}\right)=g_{\alpha}\left(\log \frac{\lambda_{0}}{\lambda_{1}}\right)
$$

where

$$
g_{\alpha}(t)=\frac{e^{\frac{\alpha}{2} t}-e^{-\frac{\alpha}{2} t}}{e^{\frac{t}{2}}-e^{-\frac{t}{2}}}, \quad t \in \mathbb{R} .
$$

It follows from Lemma 10 that $g_{\alpha}, g_{\alpha}^{\prime} \in L^{2}$ if $\alpha<1$ and therefore the function $\phi_{\alpha}$ belongs to $\mathfrak{C}$ if $\alpha<1$. On the other hand, Lemma 8 implies

$$
\frac{d}{d t}\left(H_{t}^{\alpha}\right)=\lim _{\Delta t \rightarrow 0} \frac{H_{t+\Delta t}^{\alpha}-H_{t}^{\alpha}}{\Delta t}=T_{\phi_{\alpha}, t}\left(D_{t}\right)
$$

where $T_{\phi_{\alpha}, t}$ is the double operator integral with respect to the spectral measures of $H_{t}$. Since $\phi_{\alpha} \in$ $\mathfrak{A}$ and thus $T_{\phi_{\alpha}, t}$ is bounded (see Lemma 1(ii)), it now follows that ${ }^{\|}$

$$
\left\|\frac{d}{d t}\left(H_{t}^{\alpha}\right)\right\|_{\frac{p}{\alpha}} \leq c_{\alpha}\left\|H_{t}^{-\delta}\left(H_{1}-H_{0}\right) H_{t}^{-\delta}\right\|_{\frac{p}{\alpha}} .
$$

\footnotetext{
"The estimate (4.4) in [3] is proved via usage of the integral representation of fractional powers.
} 
Recall that $G=H_{1}-H_{0}$ is positive and $H_{0}$ is positive. So,

$$
H_{t}=H_{0}+t G \geq t G
$$

Using again the fact that $t \mapsto t^{2 \delta}$ is operator-monotone, it now follows

$$
t^{2 \delta} G^{2 \delta} \leq H_{t}^{2 \delta} \Rightarrow H_{t}^{-\delta} G^{2 \delta} H_{t}^{-\delta} \leq t^{-2 \delta} \Rightarrow\left\|G^{\delta} H_{t}^{-\delta}\right\|=\left\|H_{t}^{-\delta} G^{2 \delta} H_{t}^{-\delta}\right\|^{\frac{1}{2}} \leq t^{-\delta} .
$$

Together with (4.4) it implies that

$$
\begin{aligned}
\left\|\frac{d}{d t}\left(H_{t}^{\alpha}\right)\right\|_{\frac{p}{\alpha}} \leq c_{\alpha}\left\|H_{t}^{-\delta} G H_{t}^{-\delta}\right\|_{\frac{p}{\alpha}} & \\
\leq\left\|H_{t}^{-\delta} G^{\delta} G^{\alpha} G^{\delta} H_{t}^{-\delta}\right\|_{\frac{p}{\alpha}} \leq c_{\alpha} t^{-2 \delta}\left\|G^{\alpha}\right\|_{\frac{p}{\alpha}} & =c_{\alpha} t^{\alpha-1}\left\|H_{1}-H_{0}\right\|_{p}^{\alpha} .
\end{aligned}
$$

Hence, we finish the proof by noting

$$
\begin{aligned}
\left\|H_{1}^{\alpha}-H_{0}^{\alpha}\right\|_{\frac{p}{\alpha}}=\| \int_{0}^{1} \frac{d}{d t}\left(H_{t}^{\alpha}\right) & \|_{\frac{p}{\alpha}} \\
& \leq c_{\alpha}\left\|H_{1}-H_{0}\right\|_{p}^{\alpha} \int_{0}^{1} t^{\alpha-1} d t
\end{aligned}
$$$$
=\frac{c_{\alpha}}{\alpha}\left\|H_{1}-H_{0}\right\|_{p}^{\alpha}
$$

The lemma is completely proved.

Remark 17. Similarly to Remark 13, the result above has the following submajorisation version

$$
H_{1}^{\alpha}-H_{0}^{\alpha} \prec \prec c_{\alpha}\left|H_{1}-H_{0}\right|^{\alpha}
$$

\section{References}

[1] T. Ando. Comparison of norms $\||f(A)-f(B)|||$ and $\||f(|A-B|)|||$. Math. Z., 197 (1988), No. 3, 403-409.

[2] N. A. Azamov, A. L. Carey, P. G. Dodds, F. A. Sukochev. Operator integrals, spectral shift, and spectral flow. Canad. J. Math., 61 (2009), No. 2, 241-263.

[3] M. Š. Birman, L. S. Koplienko, M. Z. Solomjak. Estimates of the spectrum of a difference of fractional powers of selfadjoint operators. Izv. Vysš. Učebn. Zaved. Matematika, 154 (1975), No. 3, 3-10. 
[4] M. S. Birman, M. Z. Solomyak. Double Stieltjes operator integrals. Problemy Mat. Fiz., (1966), No. 1, 33-67 (Russian).

[5] M. S. Birman, M. Z. Solomyak. Double Stieltjes operator integrals, II. Problemy Mat. Fiz., (1967), No. 2, 26-60 (Russian).

[6] M. S. Birman, M. Z. Solomyak. Double Stieltjes operator integrals, III. Problemy Mat. Fiz., (1973), No. 6, 27-53 (Russian).

[7] Yu. L. Daleckiǔ, S.G. Kreĭn. Formulas of differentiation according to a parameter of functions of Hermitian operators. Doklady Akad. Nauk SSSR (N.S.), 76 (1951), 13-16 (Russian).

[8] Yu. L. Daleckiŭ, S.G. Krel̆n. Integration and differentiation of functions of Hermitian operators and applications to the theory of perturbations. Voronež. Gos. Univ. Trudy Sem. Funkcional. Anal., 1 (1956), 81-105 (Russian).

[9] A. L. Carey, D. S. Potapov, F. A. Sukochev. Spectral flow is the integral of one forms on Banach manifolds of self adjoint Fredholm operators. Adv. Math, 222 (2009), 1809-1849.

[10] A. Connes, H. Moscovici. Transgression du caractère de Chern et cohomologie cyclique. C. R. Acad. Sci. Paris Sér. I Math., 303 (1986), No. 18, 913-918.

[11] P. G. Dodds, T. K. Dodds. On a submajorization inequality of T. Ando. Operator theory in function spaces and Banach lattices, Oper. Theory Adv. Appl., 75, (1995), 113-131.

[12] P. G. Dodds, F.A. Sukochev. Submajorisation inequalities for convex and concave functions of sums of measurable operators. Positivity, 13 (2009), No. 1, 107-124.

[13] I.C. Gohberg. M.G. Krel̆n. Introduction to the theory of linear nonselfadjoint operators. Translations of Mathematical Monographs, Providence, R.I., AMS, 18, 1969.

[14] N.J. Kalton, F.A Sukochev. Symmetric norms and spaces of operators. J. Reine Angew. Math., 621 (2008), 81-121.

[15] H. Kosaki. Positive definiteness of functions with applications to operator norm inequalities. Preprint, 2009.

[16] B. de Pagter, F. A. Sukochev. Differentiation of operator functions in non-commutative $L_{p^{-}}$ spaces. J. Funct. Anal., 212 (2004), No. 1, 28-75.

[17] B. de Pagter, F. A. Sukochev. Commutator estimates and $\mathbb{R}$-flows in non-commutative operator spaces. Proc. Edinb. Math. Soc., 50 (2007), No. 2, 293-324.

[18] B. de Pagter, F. A. Sukochev, H. Witvliet. Double operator integrals. J. Funct. Anal., 192 (2002), No. 1, 52-111.

[19] F. Gesztesy, A. Pushnitski, B. Simon. On the Koplienko spectral shift function. I. Basics. Zh. Mat. Fiz. Anal. Geom., 4 (2008), No. 1, 63-107, 202. 
[20] E. Heinz. Beiträge zur Störungstheorie der Spektralzerlegung. Math. Ann., 123 (1951), 415438.

[21] A. McIntosh. Heinz inequalities and perturbation of spectral families. Macquarie Mathematical Reports, 79-0006 (1979).

[22] G. Pisier, Q. Xu. Non-commutative $L_{p}$-spaces. Handbook of the geometry of Banach spaces, Vol. 2, North-Holland, Amsterdam, 2003, 1459-1517.

[23] D. Potapov, F. Sukochev. Lipschitz and commutator estimates in symmetric operator spaces. J. Operator Theory, 59 (2008), No. 1, 211-234.

[24] D. Potapov, F. Sukochev. Unbounded Fredholm modules and double operator integrals. J. Reine Angew. Math., 626 (2009), 159-185.

[25] I.E. Segal. A non-commutative extension of abstract integration. Annals of Mathematics, 57 (1953), 401-457.

[26] B. Simon. Trace ideals and their applications. Mathematical Surveys and Monographs, AMS, Providence, RI, 120 (2005).

[27] F. A. Sukochev, V. I. Chilin. The triangle inequality for operators that are measurable with respect to Hardy-Littlewood order. Izv. Akad. Nauk UzSSR Ser. Fiz.-Mat. Nauk, (1988), No. 4, 44-50 (Russian).

[28] F. A. Sukochev, V. I. Chilin. Symmetric spaces over semifinite von Neumann algebras. Soviet Math. Dokl., 42 (1991), No. 1, 97-101 (Russian).

[29] F. A. Sukochev, V. I. Chilin. Weak convergence in non-commutative symmetric spaces. J. Operator Theory, 31 (1994), No. 1, 35-65.

[30] J. von Neumann. Some matrix inequalities and metrization of matric-space. Rev. Tomsk Univ., 1 (1937), 286-300. 